\title{
Association of Serum Adropin Concentrations with Diabetic Nephropathy
}

\author{
Wenchao $\mathrm{Hu}^{1}$ and $\mathrm{Li} \mathrm{Chen}^{2}$ \\ ${ }^{1}$ Department of Endocrinology, Qilu Hospital, Shandong University, Qingdao, China \\ ${ }^{2}$ Department of Endocrinology, Qilu Hospital, Shandong University, 107 West Wenhua Road, Jinan, Shandong 250012, China
}

Correspondence should be addressed to Li Chen; chenli3@medmail.com.cn

Received 27 April 2016; Revised 26 June 2016; Accepted 29 June 2016

Academic Editor: Ariadne Malamitsi-Puchner

Copyright (c) 2016 W. Hu and L. Chen. This is an open access article distributed under the Creative Commons Attribution License, which permits unrestricted use, distribution, and reproduction in any medium, provided the original work is properly cited.

\begin{abstract}
Objective. Adropin is a newly identified regulatory protein encoded by the Enho gene and is critically involved in energy homeostasis and insulin sensitivity. This study aims to determine the correlation of serum adropin concentrations with diabetic nephropathy (DN). Methods. This study consisted of 245 patients with type 2 diabetes mellitus (T2DM) and 81 healthy subjects. Then T2DM patients were divided into normoalbuminuria, microalbuminuria, and macroalbuminuria subgroups based on urine albumin to creatinine ratio (ACR). Results. T2DM patients showed significantly lower serum adropin concentrations than those in the controls. T2DM patients with macroalbuminuria had significantly decreased serum adropin concentrations compared with the other three groups. In addition, T2DM patients with microalbuminuria showed lower serum adropin concentrations than those in patients with normoalbuminuria. Logistic regression analysis showed that serum adropin was correlated with decreased risk of developing T2DM and DN. Pearson correlation analysis indicated that serum adropin was negatively correlated with body mass index (BMI), blood urea nitrogen, creatinine, and ACR and positively correlated with glomerular filtration rate. Furthermore, multiple linear regression analysis showed that BMI and ACR were negatively correlated with serum adropin levels. Conclusion. Serum adropin concentrations are negatively associated with renal function. Adropin may be implicated in the pathogenesis of DN development.
\end{abstract}

\section{Introduction}

Diabetic nephropathy (DN), which is the leading cause of end-stage kidney disease, occurs in about $20-40 \%$ of diabetic patients [1]. Traditionally, metabolic and hemodynamic alterations caused by hyperglycemia and hypertension could contribute to renal injury in diabetic patients [2]. No effective treatments have been developed for DN. Currently available treatment options can only delay DN progression or turn to renal replacement therapies. Therefore, it is urgent to identify novel biomarkers for early diagnosis and develop some effective treatment strategies for patients at high risk.

Adropin is a newly identified metabolic hormone expressed in the liver and brain of mice [3]. Adropin is involved in the mechanism of increased adiposity, insulin resistance, and glucose and lipid metabolism [3]. Treatment with adropin can decrease blood glucose and improve insulin resistance in streptozotocin-induced type 2 diabetic rats [4]. It is reported that serum adropin levels decreased in patients with type 2 diabetes mellitus (T2DM) [5]. These findings indicate that adropin may be involved in the mechanism of T2DM. Adropin expression levels also increased in the kidney tissues of rats with streptozotocin-induced experimental diabetes [6]. Therefore, adropin may play a role in the pathogenesis of DN.

This study aims to determine the association of serum adropin with the development and progression of DN.

\section{Materials and Methods}

2.1. Patients. A total of 245 patients with T2DM were enrolled in this study. The patients were all diagnosed with T2DM in accordance with the American Diabetic Association criteria. T2DM patients were divided into three groups based on urine albumin to creatinine ratio (ACR): normoalbuminuria group (ACR < $30 \mathrm{mg} / \mathrm{g} ; n=110$ ), microalbuminuria group (30 $\leq \mathrm{ACR} \leq 300 \mathrm{mg} / \mathrm{g} ; n=95)$, and macroalbuminuria group (ACR > $300 \mathrm{mg} / \mathrm{g} ; n=40$ ). Subjects were excluded 
if they had severe cardiovascular diseases, malignant tumor, acute infection, or endocrine diseases. All patients received antidiabetic drug to control glycemia and were responsive to their respective therapies. The control group consisted of 81 healthy subjects who visited the general health check-up center of our hospital. None received medication or dietary supplements. And all the control subjects had no history of diabetes.

This study was approved by the hospital ethics board and all patients provided written informed consent.

2.2. Measurements. Serum was obtained from blood samples by centrifugation and stored at $-80^{\circ} \mathrm{C}$ until analysis. Serum adropin concentrations were measured using an enzymelinked immunosorbent assay kit (Phoenix Pharmaceuticals, Inc., USA) (coefficients of variations (CVs) for intra-assay: 5-7\%; CVs for interassay: $12-15 \%$; sensitivity: $0.3 \mathrm{ng} / \mathrm{mL}$, detection limit range: $0.01-100 \mathrm{ng} / \mathrm{mL}$ ). Metabolic syndrome (MetS) was defined using the criteria established in the third report of the National Cholesterol Education Program Expert Panel on Detection, Evaluation, and Treatment in Asian population (Adult Treatment Panel III) [7].

2.3. Statistical Analysis. Sample size was determined through power analysis using preliminary data obtained in our laboratory with the following assumptions: $\alpha$ of 0.05 (twotailed) and power of $90 \%$. A minimum of 11 subjects in the three T2DM subgroup and control group allowed the detection of difference in serum adropin concentrations. Data were expressed as means \pm standard deviation or median (interquartile range). The differences of characteristics between three groups of T2DM patients and control subjects were compared using Chi-square tests, one-way ANOVA, or Kruskal-Wallis test. Logistic regression analysis was used to determine the risk factors for developing T2DM and DN. The correlations between serum adropin and other parameters were analyzed by Pearson correlation analysis. Multiple linear regression analysis was used to determine the contribution of various factors to serum adropin. $P$ values less than 0.05 were considered statistically significant.

\section{Results}

3.1. Baseline Clinical Characteristics of T2DM Patients and Controls. The clinical parameters of T2DM patients and healthy controls are displayed in Table 1. T2DM patients showed elevated levels of systolic blood pressure (SBP), $\mathrm{HbAlc}$, and the prevalence of MetS, as well as decreased levels of high-density lipoprotein cholesterol (HDL-C) compared with control subjects. In addition, significantly higher levels of blood urea nitrogen (BUN) and creatinine (Cr) and lower levels of glomerular filtration rate (GFR) were found in T2DM patients with macroalbuminuria compared with the other three groups.

3.2. Serum Adropin Concentrations. As shown in Table 1, serum adropin concentrations were significantly elevated in the control group compared with T2DM patients. Serum adropin concentrations were significantly reduced in T2DM patients with macroalbuminuria compared with those with normoalbuminuria and microalbuminuria. In addition, T2DM patients with microalbuminuria showed lower serum adropin concentrations than those with normoalbuminuria.

3.3. The Association of Serum Adropin Concentrations with T2DM. T2DM patients showed decreased serum adropin concentrations compared with healthy controls (2.88 (2.43$3.41) \mathrm{ng} / \mathrm{mL}$ versus $3.71(2.82-4.56) \mathrm{ng} / \mathrm{mL}, P<0.001)$. As presented in Table 2, logistic regression analysis showed that serum adropin was negatively correlated with T2DM (OR 0.282 , 95\% CI 0.195 to $0.406 ; P<0.001)$. After adjusting for age and gender, serum adropin was associated with a decreased risk of developing T2DM (OR 0.274, 95\% CI 0.190 to $0.396 ; P<0.001)$. Simple logistic regression analysis showed that SBP, diastolic blood pressure (DBP), HDLC, low-density lipoprotein cholesterol (LDL-C), BUN, the prevalence of MetS, and serum adropin showed a trend $(P<$ 0.05 ) toward an association with T2DM. All of these variables were then entered into a backward stepwise multivariate logistic regression model. Multivariate logistic regression revealed that serum adropin remained a significant predictor of T2DM (OR $0.278,95 \%$ CI 0.160 to $0.485 ; P<0.001$ ).

3.4. The Association of Serum Adropin Concentrations with DN. T2DM patients with microalbuminuria and macroalbuminuria were considered to have DN. Lower serum adropin concentrations were found in T2DM patients with DN compared with those without DN (2.73 (2.24-3.10) ng/mL versus 3.17 (2.63-3.68) ng/mL, $P<0.001)$. As shown in Table 3, logistic regression analysis showed that serum adropin was inversely associated with DN development (OR 0.288, 95\% CI 0.183 to $0.453 ; P<0.001)$. After adjusting for age and gender, serum adropin was still negatively correlated with the risk of developing DN (OR 0.285, 95\% CI 0.180 to 0.452 ; $P<0.001$ ). Simple logistic regression analysis showed that SBP, DBP, total cholesterol (TC), LDL-C, BUN, Cr, GFR, and serum adropin showed a trend $(P<0.05)$ toward an association with T2DM. All of these variables were then entered into a backward stepwise multivariate logistic regression model. Serum adropin remained a significant predictor of DN after the multivariate logistic regression (OR 0.270, 95\% CI 0.160 to $0.455 ; P<0.001)$.

3.5. The Association of Serum Adropin Concentrations with Other Clinical Characteristics. As presented in Table 4, Pearson correlation analysis showed that serum adropin was correlated with body mass index (BMI) $(r=-0.215, P=$ $0.001)$, BUN $(r=-0.245, P=0.001), \operatorname{Cr}(r=-0.285$, $P<0.001)$, ACR $(r=-0.358, P<0.001)$, and GFR $(r=$ $0.212, P<0.001)$. Then a significant correlation of serum adropin with BMI $(r=-0.210, P=0.001)$, BUN $(r=-0.219$, $P=0.001), \operatorname{Cr}(r=-0.256, P<0.001), \operatorname{ACR}(r=-0.352, P<$ $0.001)$, and GFR ( $r=0.173, P=0.007)$ was observed after adjusting for age and gender. Then we performed a multiple linear regression analysis. It showed that BMI $(\beta=-0.198$, $P=0.001)$ and ACR $(\beta=-0.300, P<0.001)$ were still negatively correlated with the serum adropin. 
TABLE 1: Clinical characteristics of T2DM patients and controls.

\begin{tabular}{|c|c|c|c|c|c|}
\hline & Control & & T2DM patic & & \\
\hline & Contiot & Normoalbuminuria & Microalbuminuria & Macroalbuminuria & $P$ \\
\hline$N$ & 81 & 110 & 95 & 40 & \\
\hline Age (years) & $58.35 \pm 7.80$ & $57.52 \pm 11.33$ & $58.45 \pm 13.16$ & $59.92 \pm 10.78$ & 0.701 \\
\hline Gender (M/F) & $46 / 35$ & $58 / 521$ & $49 / 46$ & $21 / 19$ & 0.911 \\
\hline $\mathrm{BMI}\left(\mathrm{Kg} / \mathrm{m}^{2}\right)$ & $25.73 \pm 2.84$ & $26.06 \pm 3.99$ & $26.50 \pm 3.52$ & $26.33 \pm 3.07$ & 0.512 \\
\hline $\mathrm{SBP}(\mathrm{mmHg})$ & $122.52 \pm 11.28$ & $135.50 \pm 22.26^{\mathrm{a}}$ & $147.63 \pm 29.95^{\mathrm{ab}}$ & $156.65 \pm 23.26^{\mathrm{abc}}$ & $<0.001$ \\
\hline $\mathrm{DBP}(\mathrm{mmHg})$ & $79.10 \pm 7.45$ & $80.45 \pm 14.26$ & $88.00 \pm 19.60^{\mathrm{ab}}$ & $86.08 \pm 12.74^{\mathrm{ab}}$ & 0.001 \\
\hline HbAlc (\%) & $4.76 \pm 0.32$ & $7.85 \pm 1.43^{\mathrm{a}}$ & $8.06 \pm 1.23^{\mathrm{a}}$ & $7.96 \pm 1.67^{\mathrm{a}}$ & $<0.001$ \\
\hline $\mathrm{TG}(\mathrm{mmol} / \mathrm{L})$ & $2.08 \pm 1.58$ & $1.89 \pm 1.10$ & $2.16 \pm 1.92$ & $2.05 \pm 1.11$ & 0.626 \\
\hline $\mathrm{TC}(\mathrm{mmol} / \mathrm{L})$ & $5.23 \pm 0.90$ & $5.09 \pm 1.07$ & $5.45 \pm 1.26^{\mathrm{b}}$ & $5.32 \pm 1.02$ & 0.115 \\
\hline HDL-C (mmol/L) & $1.49 \pm 0.25$ & $1.12 \pm 0.24^{\mathrm{a}}$ & $1.13 \pm 0.21^{\mathrm{a}}$ & $1.17 \pm 0.33^{\mathrm{a}}$ & $<0.001$ \\
\hline LDL-C (mmol/L) & $3.25 \pm 0.54$ & $3.39 \pm 0.89$ & $3.69 \pm 1.03^{\mathrm{a}}$ & $3.52 \pm 0.80$ & 0.006 \\
\hline BUN (nmol/L) & $5.37 \pm 1.18$ & $5.33 \pm 1.58$ & $5.91 \pm 1.98^{\mathrm{b}}$ & $8.63 \pm 3.94^{\mathrm{abc}}$ & $<0.001$ \\
\hline $\mathrm{Cr}(\mu \mathrm{mol} / \mathrm{L})$ & $66.46 \pm 10.37$ & $65.27 \pm 18.16$ & $66.33 \pm 21.27$ & $115.47 \pm 70.72^{\mathrm{abc}}$ & $<0.001$ \\
\hline $\mathrm{ACR}(\mathrm{mg} / \mathrm{g})$ & - & $15.94 \pm 4.45$ & $93.51 \pm 80.98^{b}$ & $>300^{\mathrm{bc}}$ & $<0.001$ \\
\hline $\operatorname{GFR}\left(\mathrm{mL} / \mathrm{min} / 1.73 \mathrm{~m}^{2}\right)$ & $101.77 \pm 11.85$ & $109.90 \pm 38.96$ & $109.13 \pm 33.39$ & $70.50 \pm 35.65^{\mathrm{abc}}$ & $<0.001$ \\
\hline Adropin (ng/mL) & $3.71(2.82-4.56)$ & $3.17(2.63-3.68)^{\mathrm{a}}$ & $2.85(2.32-3.27)^{\mathrm{ab}}$ & $2.56(2.07-2.85)^{\mathrm{abc}}$ & $<0.001$ \\
\hline MetS $(\%)$ & $4(4.94 \%)$ & $73(66.36 \%)^{\mathrm{a}}$ & $68(71.56 \%)^{\mathrm{a}}$ & $33(82.5 \%)^{\mathrm{a}}$ & $<0.001$ \\
\hline
\end{tabular}

${ }^{\text {a }}$ Significant versus control subjects.

${ }^{\mathrm{b}}$ Significant versus T2DM patients with normoalbuminuria.

${ }^{\mathrm{c}}$ Significant versus T2DM patients with microalbuminuria.

T2DM, type 2 diabetes mellitus; BMI, body mass index; SBP, systolic blood pressure; DBP, diastolic blood pressure; TG, triglycerides; TC, total cholesterol; HDL-C, high-density lipoprotein cholesterol; LDL-C, low-density lipoprotein cholesterol; BUN, blood urea nitrogen; Cr, creatinine; ACR, urine albumin to creatinine ratio; GFR, glomerular filtration rate; MetS, metabolic syndrome.

TABLE 2: Logistic regression analysis for determining the risk factor of developing T2DM.

\begin{tabular}{|c|c|c|c|c|c|c|}
\hline \multirow{2}{*}{ Characteristics } & \multicolumn{2}{|c|}{ Adjusting for age and gender } & \multicolumn{2}{|c|}{ Simple logistic regression } & \multicolumn{2}{|c|}{ Multiple logistic regression } \\
\hline & OR $(95 \% \mathrm{CI})$ & $P$ & OR $(95 \% \mathrm{CI})$ & $P$ & OR $(95 \% \mathrm{CI})$ & $P$ \\
\hline Age (years) & $0.991(0.965-1.017)$ & 0.479 & $0.999(0.977-1.022)$ & 0.959 & - & - \\
\hline Gender (M/F) & $1.498(0.844-2.658)$ & 0.167 & $1.201(0.724-1.993)$ & 0.477 & - & - \\
\hline BMI $\left(\mathrm{kg} / \mathrm{m}^{2}\right)$ & - & - & $1.047(0.973-1.128)$ & 0.220 & - & - \\
\hline SBP (mmHg) & - & - & $1.054(1.036-1.072)$ & $<0.001$ & $1.087(1.040-1.135)$ & $<0.001$ \\
\hline $\mathrm{DBP}(\mathrm{mmHg})$ & - & - & $1.029(1.008-1.051)$ & 0.007 & $0.927(0.870-0.988)$ & 0.020 \\
\hline $\mathrm{TG}(\mathrm{mmol} / \mathrm{L})$ & - & - & $0.977(0.830-1.150)$ & 0.780 & - & - \\
\hline $\mathrm{TC}(\mathrm{mmol} / \mathrm{L})$ & - & - & $1.028(0.815-1.297)$ & 0.816 & - & - \\
\hline HDL-C (mmol/L) & - & - & $0.004(0.001-0.016)$ & $<0.001$ & $0.004(0.001-0.030)$ & $<0.001$ \\
\hline LDL-C (mmol/L) & - & - & $1.513(1.091-2.099)$ & 0.013 & $3.906(1.950-7.823)$ & $<0.001$ \\
\hline BUN (nmol/L) & - & - & $1.201(1.037-1.391)$ & 0.015 & $1.165(0.888-1.528)$ & 0.270 \\
\hline $\mathrm{Cr}(\mu \mathrm{mol} / \mathrm{L})$ & - & - & $1.009(0.998-1.021)$ & 0.096 & - & - \\
\hline $\operatorname{GFR}\left(\mathrm{mL} / \mathrm{min} / 1.73 \mathrm{~m}^{2}\right)$ & - & - & $1.001(0.994-1.009)$ & 0.750 & - & - \\
\hline Adropin (ng/mL) & $0.274(0.190-0.396)$ & $<0.001$ & $0.282(0.195-0.406)$ & $<0.001$ & $0.246(0.130-0.465)$ & $<0.001$ \\
\hline MetS & - & - & $47.176(16.64-133.78)$ & $<0.001$ & $12.422(3.453-44.68)$ & $<0.001$ \\
\hline
\end{tabular}

\section{Discussion}

Adropin is a newly discovered peptide correlated with energy regulation and obesity. Adropin also plays an important role in glucose metabolism and diabetes. Treatment with adropin could reduce blood glucose levels and insulin resistance and improve insulin sensitivity in a rat model of T2DM [4]. Adropin treatment enhanced glucose tolerance, ameliorated insulin resistance, and promoted the preferential use of carbohydrate over fat in diet-induced obese mice [8]. Adropin knockout mice showed increased adiposity and insulin resistance as well as dyslipidemia [9]. Maternal and fetal adropin levels in gestational diabetes mellitus (GDM) group were significantly lower than those in the control women [10]. Furthermore, patients with GDM showed significantly decreased serum adropin levels compared with the healthy controls [11]. T2DM patients exhibited relatively lower adropin levels than those of nondiabetic patients [5]. 
TABLE 3: Logistic regression analysis for determining the risk factor of developing DN.

\begin{tabular}{|c|c|c|c|c|c|c|}
\hline \multirow{2}{*}{ Characteristics } & \multicolumn{2}{|c|}{ Adjusting for age and gender } & \multicolumn{2}{|c|}{ Simple logistic regression } & \multicolumn{2}{|c|}{ Multiple logistic regression } \\
\hline & OR $(95 \% \mathrm{CI})$ & $P$ & OR $(95 \% \mathrm{CI})$ & $P$ & OR $(95 \%$ CI $)$ & $P$ \\
\hline Age (years) & $1.001(0.978-1.024)$ & 0.961 & $1.010(0.989-1.031)$ & 0.373 & - & - \\
\hline Gender (M/F) & $1.167(0.673-2.024)$ & 0.583 & $1.036(0.626-1.715)$ & 0.891 & - & - \\
\hline BMI $\left(\mathrm{kg} / \mathrm{m}^{2}\right)$ & - & - & $1.029(0.960-1.103)$ & 0.414 & - & - \\
\hline SBP $(\mathrm{mmHg})$ & - & - & $1.024(1.012-1.035)$ & $<0.001$ & $1.024(1.006-1.041)$ & 0.008 \\
\hline DBP (mmHg) & - & - & $1.030(1.011-1.048)$ & 0.001 & $1.007(0.980-1.035)$ & 0.618 \\
\hline HbAlc (\%) & - & - & $1.093(0.911-1.311)$ & 0.340 & - & - \\
\hline $\mathrm{TG}(\mathrm{mmol} / \mathrm{L})$ & - & - & $1.126(0.931-1.362)$ & 0.221 & - & - \\
\hline $\mathrm{TC}(\mathrm{mmol} / \mathrm{L})$ & - & - & $1.298(1.027-1.641)$ & 0.029 & $1.888(0.710-5.020)$ & 0.203 \\
\hline HDL-C (mmol/L) & - & - & $1.419(0.494-4.071)$ & 0.515 & - & - \\
\hline LDL-C (mmol/L) & - & - & $1.339(1.010-1.775)$ & 0.043 & $0.665(0.203-2.176)$ & 0.500 \\
\hline BUN (nmol/L) & - & - & $1.346(1.165-1.555)$ & $<0.001$ & $1.380(1.111-1.714)$ & 0.004 \\
\hline $\mathrm{Cr}(\mu \mathrm{mol} / \mathrm{L})$ & - & - & $1.016(1.005-1.028)$ & 0.004 & $1.003(0.982-1.024)$ & 0.802 \\
\hline $\operatorname{GFR}\left(\mathrm{mL} / \mathrm{min} / 1.73 \mathrm{~m}^{2}\right)$ & - & - & $0.991(0.984-0.999)$ & 0.019 & $1.010(0.998-1.021)$ & 0.095 \\
\hline Adropin (ng/mL) & $0.285(0.180-0.452)$ & $<0.001$ & $0.288(0.183-0.453)$ & $<0.001$ & $0.270(0.160-0.455)$ & $<0.001$ \\
\hline MetS & - & - & $1.506(0.865-2.621)$ & 0.148 & - & - \\
\hline
\end{tabular}

TABLE 4: The correlation between serum adropin concentrations and various parameters.

\begin{tabular}{|c|c|c|c|c|c|c|}
\hline \multirow{2}{*}{ Parameters } & \multicolumn{2}{|c|}{ Pearson correlation analysis } & \multicolumn{2}{|c|}{ Adjusting for age and gender } & \multicolumn{2}{|c|}{ Multiple regression analysis } \\
\hline & $r$ & $P$ & $r$ & $P$ & $\beta$ & $P$ \\
\hline Age (years) & -0.129 & 0.044 & & & & \\
\hline Gender (M/F) & 0.060 & 0.352 & & & & \\
\hline $\mathrm{BMI}\left(\mathrm{Kg} / \mathrm{m}^{2}\right)$ & -0.215 & 0.001 & -0.210 & 0.001 & -0.198 & 0.001 \\
\hline SBP (mmHg) & -0.069 & 0.284 & & & & \\
\hline DBP (mmHg) & -0.067 & 0.296 & & & & \\
\hline HbAlc (\%) & -0.018 & 0.781 & & & & \\
\hline $\mathrm{TG}(\mathrm{mmol} / \mathrm{L})$ & 0.072 & 0.260 & & & & \\
\hline $\mathrm{TC}(\mathrm{mmol} / \mathrm{L})$ & 0.049 & 0.444 & & & & \\
\hline HDL-C (mmol/L) & -0.026 & 0.689 & & & & \\
\hline LDL-C (mmol/L) & 0.028 & 0.668 & & & & \\
\hline BUN (nmol/L) & -0.245 & $<0.001$ & -0.219 & 0.001 & -0.029 & 0.766 \\
\hline $\mathrm{Cr}(\mu \mathrm{mol} / \mathrm{L})$ & -0.285 & $<0.001$ & -0.256 & $<0.001$ & -0.105 & 0.328 \\
\hline $\mathrm{ACR}(\mathrm{mg} / \mathrm{g})$ & -0.358 & $<0.001$ & -0.352 & $<0.001$ & -0.300 & $<0.001$ \\
\hline $\operatorname{GFR}\left(\mathrm{mL} / \mathrm{min} / 1.73 \mathrm{~m}^{2}\right)$ & 0.212 & $<0.001$ & 0.173 & 0.007 & 0.006 & 0.941 \\
\hline MetS & 0.006 & 0.921 & - & - & - & - \\
\hline
\end{tabular}

The present study demonstrated that serum adropin was correlated with decreased risk of developing T2DM after the logistic regression analysis. Hence, serum adropin should be utilized as a biomarker for assessing the risk of developing T2DM. However, no investigation has focused on the association of serum adropin with type 1 diabetes. Furthermore, we did not find a correlation of serum adropin with $\mathrm{HbAlc}$ which is a parameter for average blood glucose. Previous studies showed the role of adropin in glucose metabolism. Therefore, the association of serum adropin with blood glucose should be illustrated by future studies.

This study showed that decreased serum adropin concentrations were correlated with the development and progression of DN. Previous studies determined the role of adropin in DN. Adropin expression was detected in the kidney tissue of rats, including the glomerulus, peritubular interstitial cells, and peritubular capillary endothelial cells [6]. Adropin immunoreaction was enhanced in the kidney of diabetesinduced rats compared with that in the kidney of the controls [6]. The intensities of adropin immunoreactivity increased with diabetic severity [12]. However, the reason why serum adropin concentrations were low while adropin immunoreaction was high in diabetes or DN condition remains unknown. Further studies are required to explain this phenomenon.

The present study showed that serum adropin was correlated with renal function parameters such as BUN, Cr, and GFR. Adropin may be involved in the pathogenesis of kidney disease, not only DN. Future studies performed in patients with other kidney disease are needed to explain the precise role of adropin in kidney disease. 
The precise role of adropin in DN mechanism remains unclear. Inflammation plays an important role in the development of DN. Adropin significantly decreased the mRNA expression levels of tumor necrosis factor-alpha (TNF- $\alpha$ ) and interleukin 6 (IL-6) in the pancreas tissue of diabetic rats [4]. Circulating adropin level was negatively correlated with TNF- $\alpha$ level in women with polycystic ovarian syndrome [13]. Therefore, we hypothesize that adropin may play a protective role in DN development through anti-inflammatory effects.

We also evaluate the correlation of adropin with MetS. We found that serum adropin was correlated with BMI which is a parameter for obesity. However, no significant correlation of serum adropin with dyslipidemia, blood glucose, or blood pressure was found in the present study. Moreover, we defined MetS in the control and case groups and found that there is no correlation of serum adropin with MetS. Hence, adropin may be closely correlated with obesity. However, there is not enough evidence to conclude a correlation of adropin with MetS.

This study presents several limitations. First, the sample size was not sufficiently large to achieve definitive conclusions. Further studies with large populations are thus warranted. Second, our study utilized a cross-sectional design. Hence, the causative relation must be confirmed by future longitudinal studies.

In conclusion, serum adropin concentrations are negatively associated with renal function. Adropin may be implicated in the pathogenesis of DN development.

\section{Competing Interests}

The authors declare that they have no competing interests.

\section{References}

[1] E. Ritz, I. Rychlík, F. Locatelli, and S. Halimi, "End-stage renal failure in type 2 diabetes: a medical catastrophe of worldwide dimensions," American Journal of Kidney Diseases, vol. 34, no. 5, pp. 795-808, 1999.

[2] B. Satirapoj, "Nephropathy in diabetes," Advances in Experimental Medicine and Biology, vol. 771, pp. 107-122, 2013.

[3] K. G. Kumar, J. L. Trevaskis, D. D. Lam et al., "Identification of adropin as a secreted factor linking dietary macronutrient intake with energy homeostasis and lipid metabolism," Cell Metabolism, vol. 8, no. 6, pp. 468-481, 2008.

[4] R. Akcilar, F. E. Kocak, H. Simsek et al., "Antidiabetic and hypolipidemic effects of adropinin streoptozotocin-induced type 2 diabetic rats," Bratislava Medical Journal, vol. 117, no. 2, pp. 100-105, 2016.

[5] L. Wu, J. Fang, L. Chen et al., "Low serum adropin is associated with coronary atherosclerosis in type 2 diabetic and nondiabetic patients," Clinical Chemistry and Laboratory Medicine, vol. 52, no. 5, pp. 751-758, 2014.

[6] S. Aydin, T. Kuloglu, S. Aydin et al., "Expression of adropin in rat brain, cerebellum, kidneys, heart, liver, and pancreas in streptozotocin-induced diabetes," Molecular and Cellular Biochemistry, vol. 380, no. 1-2, pp. 73-81, 2013.

[7] Executive Summary of the Third Report of the National Cholesterol Education Program (NCEP) Expert Panel on Detection, Evaluation, and Treatment of High Blood Cholesterol in Adults
(Adult Treatment Panel III), "Expert panel on detection, evaluation, and treatment of high blood cholesterol in adults," The Journal of the American Medical Association, vol. 285, no. 19, pp. 2486-2497, 2001.

[8] S. Gao, R. P. McMillan, Q. Zhu, G. D. Lopaschuk, M. W. Hulver, and A. A. Butler, "Therapeutic effects of adropin on glucose tolerance and substrate utilization in diet-induced obese mice with insulin resistance," Molecular Metabolism, vol. 4, no. 4, pp. 310-324, 2015.

[9] K. Ganesh Kumar, J. Zhang, S. Gao et al., "Adropin deficiency is associated with increased adiposity and insulin resistance," Obesity, vol. 20, no. 7, pp. 1394-1402, 2012.

[10] E. Celik, E. Yilmaz, O. Celik et al., "Maternal and fetal adropin levels in gestational diabetes mellitus," Journal of Perinatal Medicine, vol. 41, no. 4, pp. 375-380, 2013.

[11] A. Beigi, N. Shirzad, F. Nikpour, E. Nasli Esfahani, S. Emamgholipour, and F. Bandarian, "Association between serum adropin levels and gestational diabetes mellitus; a case-control study," Gynecological Endocrinology, vol. 31, no. 12, pp. 939-941, 2015.

[12] T. Kuloglu and S. Aydin, "Immunohistochemical expressions of adropin and inducible nitric oxide synthase in renal tissues of rats with streptozotocin-induced experimental diabetes," Biotechnic \& Histochemistry, vol. 89, no. 2, pp. 104-110, 2014.

[13] T. Kume, M. Calan, O. Yilmaz et al., "A possible connection between tumor necrosis factor alpha and adropin levels in polycystic ovary syndrome," Journal of Endocrinological Investigation, vol. 39, no. 7, pp. 747-754, 2016. 


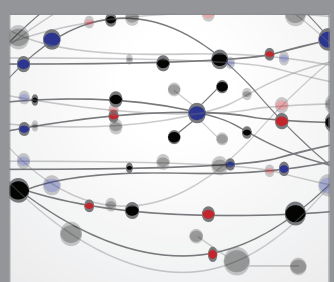

The Scientific World Journal
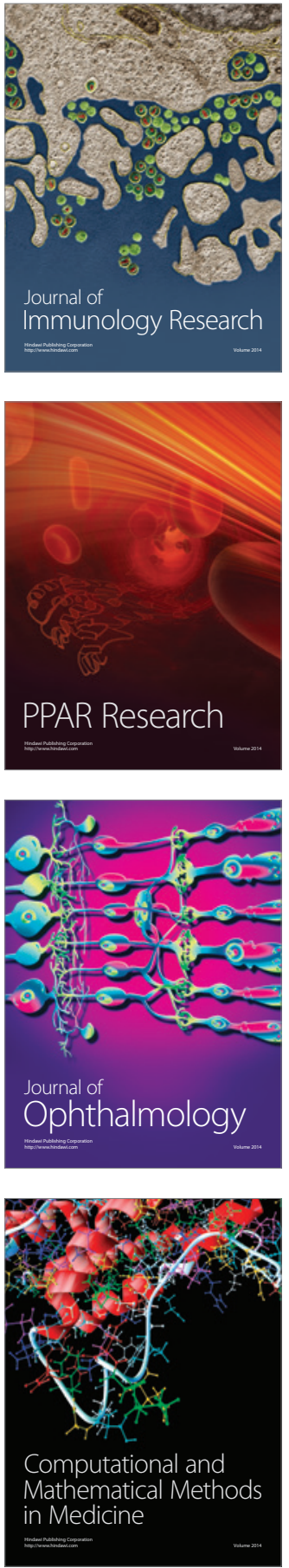

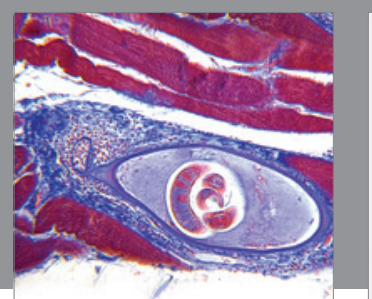

Gastroenterology Research and Practice

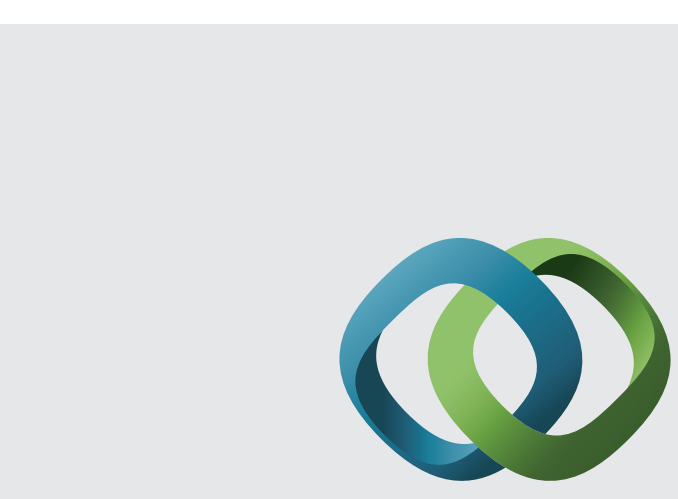

\section{Hindawi}

Submit your manuscripts at

http://www.hindawi.com
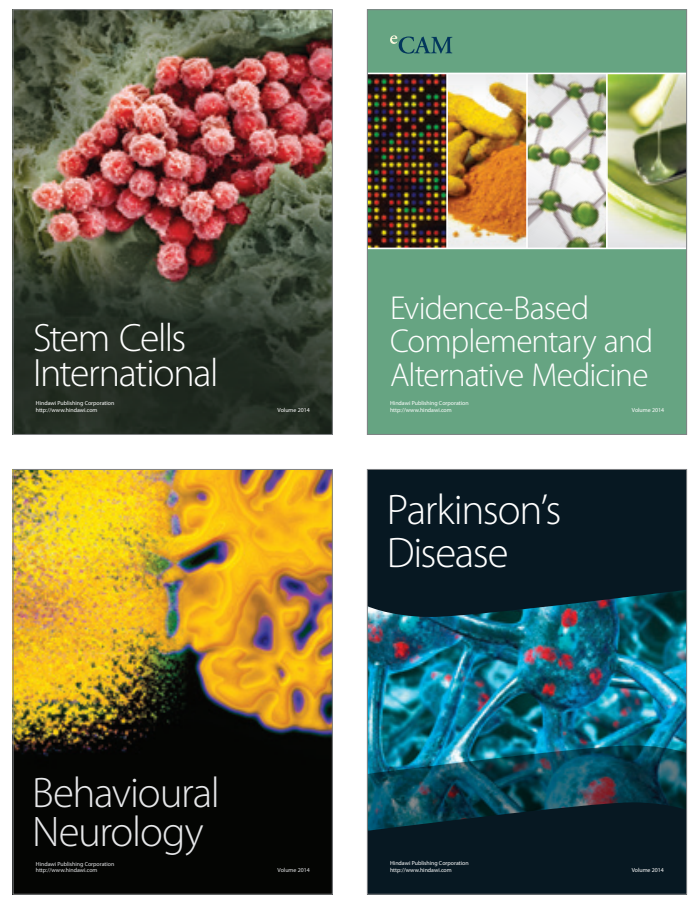
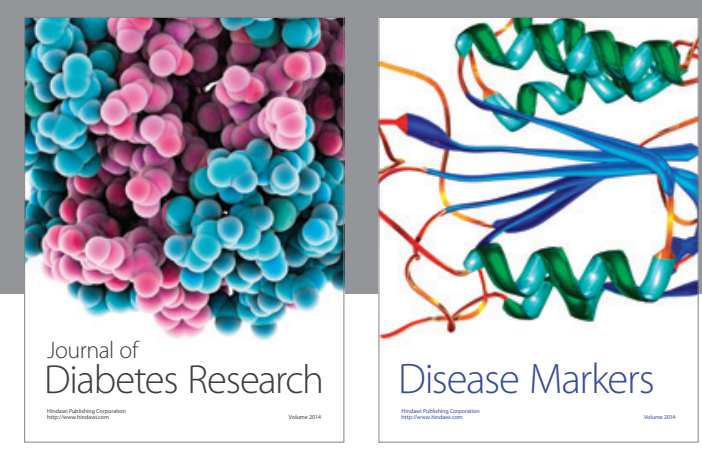

Disease Markers
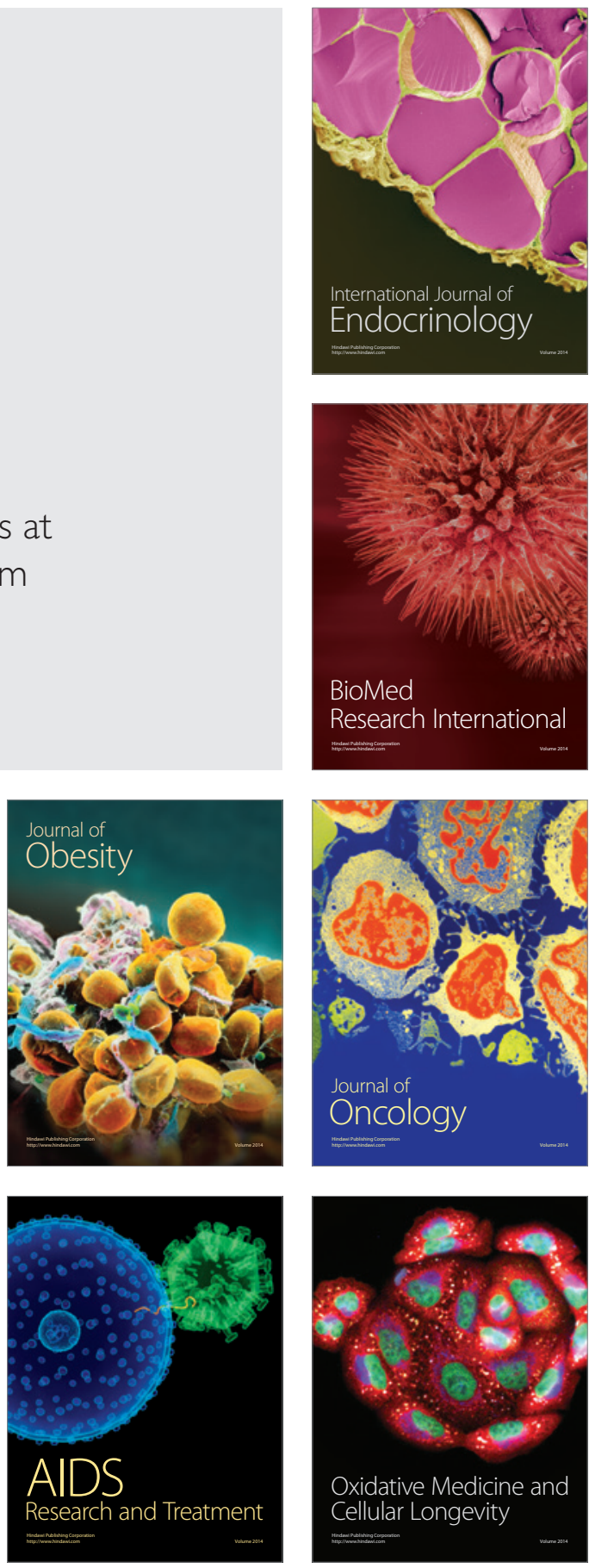